\title{
Polarization-Insensitive 5x20Gb/s WDM Ge Receiver using Compact Si Ring Filters with Collective Thermal Tuning
}

\author{
Peter De Heyn (1), Jeroen De Coster (2), Peter Verheyen (2), Guy Lepage (2), Marianna Pantouvaki (2), \\ Philippe Absil (2), Wim Bogaerts (1), Dries Van Thourhout (1) and Joris Van Campenhout (2) \\ (1) Photonics Research Group, Department of Information Technology, Ghent University - imec, B-9000 Ghent, Belgium \\ (2) Interuniversity Microelectronics Center (IMEC), Kapeldreef 75, B-3000 Leuven, Belgium \\ Peter.DeHeyn@intec.ugent.be
}

\begin{abstract}
A 5x20Gb/s WDM Ge receiver with $300 \mathrm{GHz}$ channel spacing is presented. Uniform flat-top channel responses result in a $0.1 \mathrm{~A} / \mathrm{W}$ fiber-referenced responsivity and crosstalk better than $-15 \mathrm{~dB}$ for all channels and polarization states.

OCIS codes: (130.7408) Wavelength filtering devices; (250.0040) Detectors; (250.5300) PICs
\end{abstract}

\section{Introduction}

Aggregate bandwidth requirements for I/O communication in advanced CMOS nodes and high performance applications are expected to reach the level of TB/s in the near future [1]. Optical interconnects based on silicon photonics are increasingly being considered as a viable alternative to enable further I/O scaling due to its high level of integration and potentially low power consumption and low cost. In intra-datacenter links fiber cost and front panel area are gradually becoming major driving factors to scale the bandwidth of future optical interconnects to next generation Ethernet data rates $(100 \mathrm{GbE} / 400 \mathrm{GbE} / 1.6 \mathrm{TbE})$ with the adoption of wavelength-division multiplexing (WDM) [2,3], space-division multiplexing (SDM) [4] or both [5]. Each approach has its own advantages regarding specific system requirements such as power consumption, reach, scalability and dynamic control.

For WDM enabled Si photonic transceivers, the challenges are low insertion loss (IL) and low crosstalk (XT), polarization insensitivity, compact footprint and low power consumption, manufacturability and robustness against temperature variations. As a result first-generation Si photonic WDM transceivers are likely to be based on coarse WDM with channel spacing of 20nm [6]. However, these devices will be limited in amount of channels, and are incompatible with the limited bandwidth of grating couplers. In order to increase the number of WDM channels, a more aggressive channel spacing should be pursued. In [7], cascaded lattice filters have been designed to exhibit a very broad flat transmission pass-band which is capable to overcome up to $25^{\circ} \mathrm{C}$ temperature variations without active compensation or significant filter degradation.

In this paper we use Si ring resonators to create a dense WDM (DWDM) filter with $300 \mathrm{GHz}(2.34 \mathrm{~nm})$ channel spacing around $1540 \mathrm{~nm}$ wavelength. High-confinement silicon waveguides are prone to small variation in waveguide dimensions resulting in unpredictable wavelength shifts up to $1 \mathrm{~nm}$ for local variations and up to $10 \mathrm{~nm}$ for wafer-scale variations [8] which can cause the channels to overlap with each other resulting in large XT. In [8], we have shown that the flat-top behavior of $2^{\text {nd }}$-order ring resonators can compensate for local $(10 \mu \mathrm{m}-1 \mathrm{~mm})$ fabrication variations and that the use of a collective heater can compensate for global wafer-scale variations and ambient temperature variations. Due to the compact nature of the ring resonator, this active compensation can be performed at low power when optimized, e.g. by local substrate removal among others [8] and will be easy to control (only one control pad).

In this work we extend the results of [8] by using a 2-dimensional grating coupler [9] to decouple the two orthogonal polarization states of a single mode fiber into their own five-channel $300 \mathrm{GHz}$ DWDM filter bank. Each decoupled channel is terminated with a single germanium $(\mathrm{Ge})$ high-speed lateral PIN photodiode. We demonstrate a 5-channel DWDM Ge receiver array with a highly responsive (fiber-referenced $0.1 \mathrm{~A} / \mathrm{W}$ ), polarization-insensitive and uniform channel response with crosstalk better than $-15 \mathrm{~dB}$. The photodiode signal bandwidth is larger than $20 \mathrm{GHz}$, having a $20 \mathrm{~Gb} / \mathrm{s}$ open-eye diagram at $2 \mathrm{~V}$ reverse bias while having a low dark current of $8 \mathrm{nA}$. The integrated collective heater shows uniform heating and an efficiency of $0.0247 \mathrm{~nm} / \mathrm{mW}$. In addition a high manufacturability and yield is demonstrated based on wafer-scale measurements.

\section{Design and fabrication}

The designed filter array consists of two parallel identical arms with each five cascaded second-order (racetrack) ring resonators at a channel spacing of $300 \mathrm{GHz}$ or $2.34 \mathrm{~nm}$. The ring radius is $4 \mu \mathrm{m}$ and the coupling length $6.8 \mu \mathrm{m}$ resulting in a FSR of $14 \mathrm{~nm}$. The channel spacing is achieved by a small increase in coupling length of $55 \mathrm{~nm}$ between two adjacent filters (circumference increase of $110 \mathrm{~nm}$ ). The bus-to-ring power coupling is $34 \%$ and ring-to- 
ring power of $5 \%$ which corresponds with a bus-to-ring gap of $178 \mathrm{~nm}$ and a ring-to-ring gap of $293 \mathrm{~nm}$. The wavelength dependency of the coupling is taking into account by adjusting the gap (accordingly) for each channel. The design is made on a $1 \mathrm{~nm}$ grid, an improvement compared to earlier work in [8] where a mask resolution of 5nm was used. The resulting crosstalk between the different channels is designed to be $-20 \mathrm{~dB}$. The details of the Si p++ doped heaters used can be found in [8]. A schematic of the presented polarization-diversified DWDM Ge receiver design is shown Fig.1.

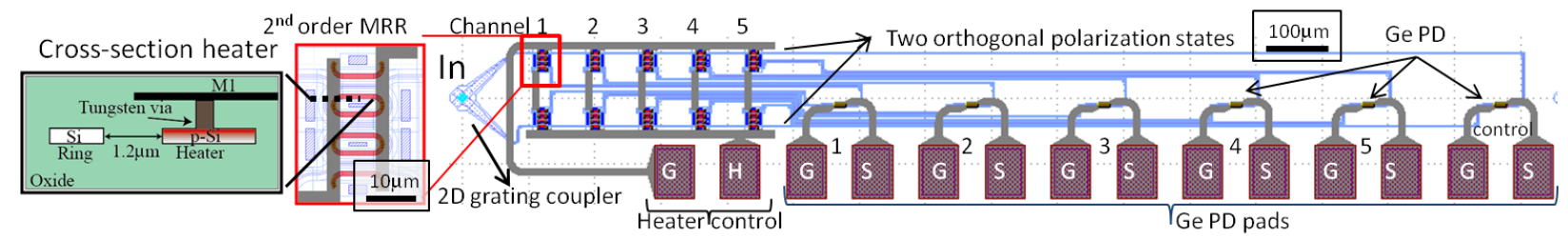

Figure 1. Schematic of a polarization-diversified DWDM receiver with five cascaded $2^{\text {nd }}$ order ring resonators, $\mathrm{p}++$ doped Si heaters and Ge PDs.

Total device footprint is $1.7 \mathrm{~mm} \times 0.28 \mathrm{~mm}$ but can easily be decreased to $1.1 \mathrm{~mm} \times 0.28 \mathrm{~mm}$ when the detector pad pitch is decreased to $50 \mu \mathrm{m}$ instead of $100 \mu \mathrm{m}$ as used now. Scaling this component to 10 channels would result in a total component size $\approx 40$ times smaller than reported in [10] for an arrayed waveguide grating based filter. Each channel is terminated with a lateral PIN germanium photodiode with an intrinsic width of $0.75 \mu \mathrm{m}$ and a length of $15 \mu \mathrm{m}$.

The WDM filter was fabricated on a $200-\mathrm{mm}$ SOI wafer with $2 \mu \mathrm{m}$ buried oxide and $220 \mathrm{~nm}$ top c-Si layer using processing modules from imec's Silicon-Photonics Platform (iSiPP) with detailed processing steps covered in [10].

\section{Measurement results}

The presented receiver is characterized using two orthogonal polarization states. The external fiber-referenced channel responsivity for each channel using a wavelength resolution of $0.1 \mathrm{~nm}$ is shown in Fig.2(a). The input $2 \mathrm{D}$ grating coupler has an insertion loss (IL) of $6.5 \mathrm{~dB}$. The germanium photodiodes have a very low dark current of around $8 \mathrm{nA}$ at $2 \mathrm{~V}$ reverse bias and a responsivity of $0.61 \mathrm{~A} / \mathrm{W}$ resulting in a reference responsivity of $0.14 \mathrm{~A} / \mathrm{W}$ (Fig.2a). The IL of the ring resonator filters is about $1.2 \mathrm{~dB}$, bringing the overall fiber-referenced responsivity to $0.1 \mathrm{~A} / \mathrm{W}$. The polarization dependence of the IL is very small $(<0.5 \mathrm{~dB})$ and the maximum crosstalk $(\mathrm{XT})$ between the 5 channels is $-15 \mathrm{~dB}$ (between channel 3 and 4). To enable collective tuning, a uniform channel spacing is necessary as explained in [8]. To illustrate this, we overlay the responses of all channels, both polarizations, according to the designed $300 \mathrm{GHz}$ or $2.34 \mathrm{~nm}$ channel spacing, as shown in Fig.2(b), showing in a channel spacing (CS) variation of $0.6 \mathrm{~nm}$. Because the $1 \mathrm{~dB}$ BW of the channel response is $1.2 \mathrm{~nm}$, a common (over all channels and polarization states) flat band of $0.6 \mathrm{~nm}$ is left over.

The bandwidth of the photodiodes is characterized using a 50GHz Lightwave Network Analyzer. The normalized transmission $\mathrm{s}_{21}$-parameters for all five channels are shown in Fig.2(e) demonstrating a $3 \mathrm{~dB}$ BW ranging between $20 \mathrm{GHz}$ and $26 \mathrm{GHz}$ at $2 \mathrm{~V}$ reverse bias. At smaller bias voltages the $\mathrm{BW}$ is measured $13.5 \mathrm{GHz}$ at $0.5 \mathrm{~V}$ and $18.5 \mathrm{GHz}$ at $1 \mathrm{~V}$ reverse bias. The same photodiode is also characterized using a pseudo-random bit sequence with a length of $2^{\wedge} 7-1$ at $20 \mathrm{~Gb} / \mathrm{s}$ at $2 \mathrm{~V}$ reverse bias. No electrical amplification is used to visualize the received signal on an oscilloscope and therefore a rather large optical input power of $11.5 \mathrm{dBm}$ is used. In Fig.2(f), the resulting eye diagram is showing a clearly open eye at $20 \mathrm{~Gb} / \mathrm{s}$.

In order to investigate the manufacturability of the device, wafer-scale measurements were performed on a purely optical test structure containing the same filter array but having two separate one-dimensional input grating couplers for TE polarization only and no Ge-detectors, as shown in Fig.2(c). In the table shown in Fig.2(d), the mean value and percentiles for the main characteristics are listed showing very uniform wafer-scale performance. The $1-\mathrm{dB}$ and $3-\mathrm{dB}$ bandwidth (BW) is tightly confined between $1.17 \mathrm{~nm}$ and $1.59 \mathrm{~nm}$. Due to a slightly different patterning process, the coupling between the waveguides on this wafer is smaller resulting in a better XT of -18.8dB and an IL of around $1.2 \mathrm{~dB}$. Collective tuning will be used to overcome wafer-scale waveguide non-uniformity which causes the channel responses to shift in center wavelength. This detrimental shift is characterized in Fig.2(h) where the center wavelength of channel 1 is plotted in function of its position on the wafer. As listed in Fig.2(d), $80 \%$ of the devices is lying within $7 \mathrm{~nm}$ of the wafer mean value of $1529 \mathrm{~nm}$ showing a good within-wafer waveguide uniformity. This good uniformity lowers the maximum required tuning range and thus the power consumption.

To characterize the integrated heaters used for collective tuning, we use the optical test structure, where an additional substrate removal step was used to improve the efficiency. The relative shift of the resonances of all five channels in function of applied power is shown in Fig. $2(\mathrm{~g})$, showing an efficiency of $0.0247 \mathrm{~nm} / \mathrm{mW} \mathrm{resulting} \mathrm{in}$ 
$142 \mathrm{~mW}$ for a full FSR (14nm) tuning, calculated per channel. This efficiency is likely to be improved by an optimizing the heater design (e.g. distance between heater and waveguide). A tight uniformity over all channels of the heater tunability is obtained, which is essential for collective thermal control.
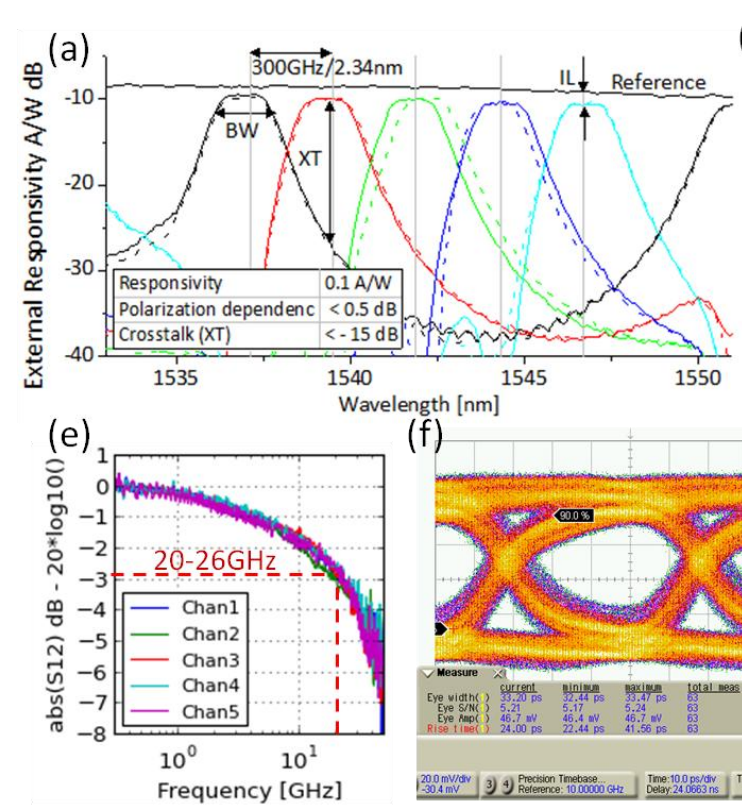

(f)

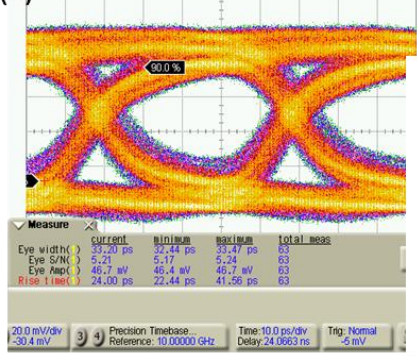

(b)

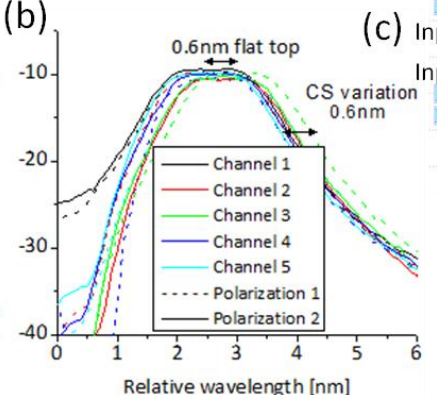

(g)

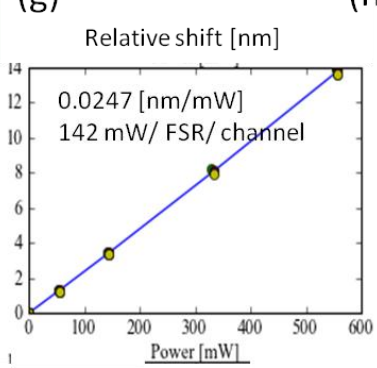

(d) (h)

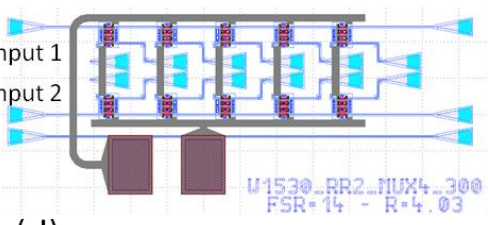

\begin{tabular}{|l|c|c|c|}
\hline Specification & Mean & P10 & P90 \\
\hline 1dB BW [nm] & 1.17 & 1.16 & 1.2 \\
3dB BW [nm] & 1.59 & 1.56 & 1.62 \\
XT at 2.4nm [dB] & -18.8 & -19.2 & -18.3 \\
IL [dB] & -1.12 & -1.5 & -0.73 \\
$\lambda 1[n m]$ & 1529 & 1525 & 1532 \\
\hline
\end{tabular}

h)

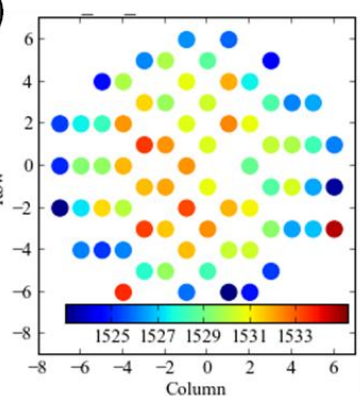

Figure 2. (a) The fiber-referenced responsivity spectrum of the as-fabricated 5-channel WDM Ge receiver for both polarizations (solid/dashed lines). (b) Overlaid channel responses showing a common flat top and a channel spacing (CS) variation of both $0.6 \mathrm{~nm}$. (c) Optical test structure used for wafer-scale manufacturability (d) Measured wafer-level statistics. Mean and both percentiles $\left(10^{\text {th }}, 90^{\text {th }}\right)$ are based on the mean value of the 5 channels (both arms) measured on (c). (e) S21-parameters of all channel PDs (f) 20Gb/s open-eye diagram with bias $-2 \mathrm{~V}$ (g) Relative peak wavelength shifts versus power applied to the collective heater. (h) Central wavelength the first channel $\left(\lambda_{1}\right)$ relative to its position on the wafer.

\section{Conclusion}

A polarization-diversified five-channel WDM Ge Receiver with a dense $300 \mathrm{GHz}$ channel spacing is presented. Flat-top $2^{\text {nd }}$-order ring resonators are used to overcome local fabrication variations resulting in a polarizationinsensitive fiber-referenced responsivity of $0.1 \mathrm{~A} / \mathrm{W}$ for all channels and XT better than $-15 \mathrm{~dB}$. An integrated collective heater can compensate wafer-scale variations and ambient temperature variations at $0.0247 \mathrm{~nm} / \mathrm{mW}$. Each channel photodiode has a bandwidth larger than $20 \mathrm{GHz}$ and exhibits an open eye diagram at $20 \mathrm{~Gb} / \mathrm{s}$. Wafer-scale measurements show very promising results in terms of manufacturability as well as scalability towards larger amount of channels and more dense channel spacing WDM receivers. We believe that improved design of the 2D grating coupler and photodetector will directly increase the fiber-referenced channel responsivity up to $0.3 \mathrm{~A} / \mathrm{W}$ and signal bandwidth up to $>50 \mathrm{GHz}$.

\section{Acknowledgement}

The authors acknowledge the efforts of imec's $200 \mathrm{~mm}$ p-line and DTE team for their contributions to the device fabrication and mask tape-out. This work is supported by the ERC project ULPPIC and by imec's Core Partner Program.

\section{References}

[1] D. A. B. Miller, "Device Requirements for Optical Interconnects to Silicon Chips," Proceedings of the IEEE, vol. 97, no. 7, 2009.

[2] C. R. Doerr, et.al, "Eight-Channel SiO/SiN/Si/Ge CWDM Receiver," IEEE PTL, vol. 23, no. 17, pp. 1201-1203, 2011.

[3] L. Chen, et.al., IEEE Photonics Technology Letters, vol. 23, no. 13, pp. 869-871, Jul. 2011.

[4] A. M. J. Koonen, "Silicon Photonic Integrated Mode Multiplexer and Demultiplexer," IEEE PTL, vol. 24, no. 21, pp. 1961-1964, 2012.

[5] C. R. Doerr and T. F. Taunay, IEEE Photonics Technology Letters, vol. 23, no. 9, pp. 597-599, 2011.

[6] http://www.ieee802.org/3/bm/public/jul13/shen 01a 0713 optx.pdf

[7] F. Horst, et.al., Optics express, vol. 21, no. 10, pp. 11652-11658, 2013.

[8] P. De Heyn, et.al.,Journal of Lightwave Technology, vol. 31, no. 16, pp. 3085-3092, 2013.

[9] D. Taillaert, et.al. IEEE Photonics Technology Letters, vol. 15, no. 9, pp. 1249-1251, 2003.

[10] L. Chen, C. R. Doerr, and Y. Chen, OFC/NFOEC 2012, p.OW3G7.

[11] P. Verheyen et al., Proc. SPIE 8431, 843114 (2012). 